\title{
Contingent associations and the double-function, verbal-discrimination task
}

\author{
BENTON J. UNDERWOOD and CHARLES S. REICHARDT \\ Northwestern University, Evanston, Illinois 60201
}

\begin{abstract}
Three experiments examined the role of contingent associations in learning double-function, verbal-discrimination lists. Some 15-pair lists of category instances were constructed in such a way that the learning of three contingent associations based on category names would mediate correct performance for all 15 pairs. The first experiment gave no evidence that the three contingencies aided learning. The second experiment showed that subjects could be taught the three contingent associations in isolation and that they could then apply them successfully to the double-function list. To make the contingencies more apparent to the subjects, the category names were used during feedback in the third experiment. The learning was not facilitated by such feedback. It was concluded that the learning observed for the usual double-function list does not involve contingent associations.
\end{abstract}

In the double-function, verbal-discrimination list, each item is used twice, being 'correct in one pair, incorrect in another. The subject's task is to learn to identify the correct member of each pair. The interest in the performance on such lists extends into two domains. The first concerns the theoretical accounts of verbal-discrimination learning. Kausler and Boka (1968) were apparently the first to use double-function lists. They noted that the discrimination between the correct and incorrect members in a pair in a double-function list could not possibly be based on a frequency difference and, therefore, frequency theory (Ekstrand, Wallace, \& Underwood, 1966) would predict that the list could not be learned. The results presented by Kausler and Boka show that some learning did occur, albeit at a very slow pace. On the 15th trial for the 16-pair list, the mean number of correct responses was about 11 , with eight being expected by chance. The usual verbal-discrimination list (single function) was readily learned. The enormous discrepancy in difficulty between single- and double-function lists is, as expected, by frequency theory although any other theoretical approach would probably include the same expectation. Of course, any theory must sooner or later account for the fact that some learning occurs in the double-function list.

The second point of interest, following directly from the first, has to do with the mechanisms involved in the slow learning which does occur in the double-function list. One possibility, which is the focus of the current report, is that a subject learns contingent associations,

This research was supported by the Personnel and Training Research Programs, Psychological Sciences Division, Office of Naval Research, under Contract N00014-67-A-0356-0010, Contract Authority Identification NR 154-321. Reproduction in whole or in part is permitted for any purpose of the United States Government.

Requests for reprints should be sent to Benton J. Underwood, Department of Psychology, Northw estern University, Evanston, Illinois 60201 . e.g., he learns that when $A$ and $B$ occur together, $A$ is correct, but when B and C occur together, B is correct. Wickelgren (1969), in his examination of possible associative explanations for serial ordering, proposed contingent associations as one possibility. He rejected the notion on the grounds that learning contingent associations in any number was impossible or, at best, very difficult. This conclusion seems to be supported by the work of Mueller, Kausler, Yadrick, and Pavur (1974). These investigators instructed subjects in the use of contingent associations in learning a double-function list. They report that "... this strategy seems to demand too much of subjects in terms of memory load, due to the need to organize the list in terms of paired pairs."

These observations indicate a severe limitation on the memory system, a limitation either in acquiring the information needed to respond on the basis of contingencies, or in utilizing the information to mediate correct performance, or both. The present studies were designed to explore the potential of contingent associations more thoroughly. The double-function, verbal-discrimination task is peculiarly appropriate for asking about the acquisition of contingent associations. The subject rather quickly discovers that a simple rule (e.g., a frequency rule) will not produce correct responding. Under these circumstances, he should search for other rules which would apply to more than one of the pairs. We attempted to make this search easy by forming 15-pair lists in which only three contingency rules could, if discovered and implemented, provide correct responding for all 15 pairs. This was accomplished by using the names of five sports, five fruits, and five pieces of furniture. The pairings in the critical list contained three categorical contingencies, namely, given a pairing of a piece of furniture and a fruit, the furniture instance was always correct; given a fruit and a sport instance paired, the fruit instance was always correct, and given a pairing of a sport and a piece of furniture, the sport instance was always correct. 


\section{EXPERIMENT I}

\section{Method}

Lists. The following 15 pairs composed the critical list: chair-apple, bed-pear, desk-peach, dresser-cherry, bureau-lemon, apple-football, pear-basketball, peach-swimming, cherry-golf, lemon-bowling, football-chair, basketball-bed, swimming-desk, golf-dresser, bowling-bureau. For the first five pairs, the correct members were the furniture instances, for the second five pairs, the correct members were the fruit instances, and for the last five pairs, the names of the sports were correct. This three-rule contingency list will be called List $\mathrm{C}$. The noncontingent, double-function list (List NC) was constructed from the 15 words, each word being both correct and incorrect. There were no consistent contingencies between all instances of a concept and all those of another. The third list was a single-function (SF) list. Ten instances of each concept were used for the SF list, with the pairings never consisting of two instances from the same concept.

Procedure and subjects. The lists were presented at $2: 2-\mathrm{sec}$ rate for anticipation learning. During the feedback interval, the pair was shown to the left, with the correct word from that pair shown on the right. During the anticipation interval, the words in a pair were printed one above the other, with this positioning varying from trial to trial. There were three different random orders of the pairs. Following an initial study trial, 15 anticipation trials were given with the subjects instructed to respond to each pair on each trial. The subjects were not informed about the contingencies present in List $\mathrm{C}$. Twenty undergraduate students were assigned to each list following a block-randomized schedule of the three lists.

\section{Results and Discussion}

The mean numbers of errors on each of the 15 trials are shown in Figure 1. The first obvious fact is that the SF list was acquired far more rapidly than either of the two double-function lists. Yet, the performance on both List $\mathrm{C}$ and List $\mathrm{NC}$ shows improvement over trials. If the three-rule contingencies aided learning, the performance improvement should have been greater for List $\mathrm{C}$ than for List NC. The data give no indication of this; in fact, it appears that on the later trials the subjects given List NC were somewhat superior to those given List C. An analysis of the scores, including trials on the two lists as a variable, showed that overall the two groups did not differ, $F(1,38)=1.94, p>.05$. The interaction between trials and lists was of borderline significance, $F(14,532)=1.84, p<.05$.

If it is assumed that the learning of the $C$ list would have been facilitated (compared with the NC list) had the three contingencies been discovered, learned, and implemented, it must be concluded that these steps did not all occur. Thus, it would appear that performance based on contingent associations is not easy to demonstrate, a conclusion reached by other investigators, as has been noted earlier. We were not as yet prepared to accept this conclusion. Two other experiments were conducted, the first of the two being more or less preliminary to the final one.

\section{EXPERIMENT II $^{1}$}

The aim of this study was to see if subjects could be taught the three contingency rules holding for List $\mathrm{C}$, and, if so, could they be used subsequently in the performance on List $\mathbf{C}$.

\section{Method}

Training. The 16 subjects were first given a card on which the following three rules appeared: If you see Furniture and Fruit, Furniture is correct. If you see Fruit and Sport, Fruit is correct. If you see Sport and Furniture, Sport is correct. The subject was

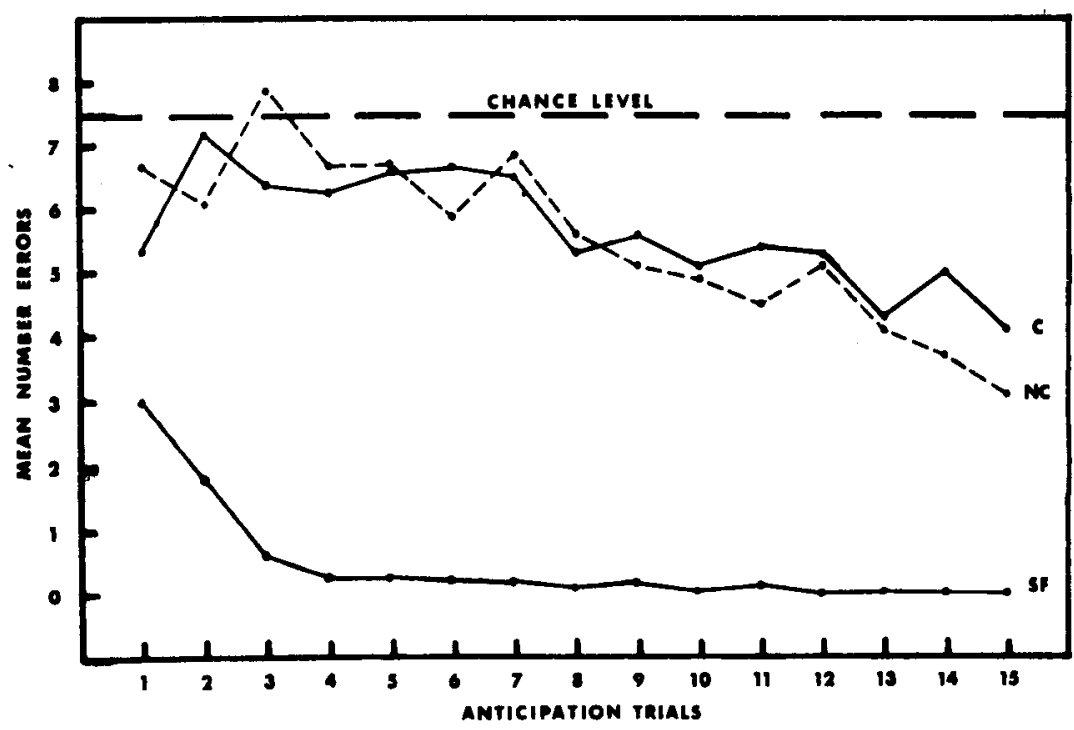

Figure 1. Mean number of errors on each of the 15 trials for the two double-function lists $(\mathrm{C}, \mathrm{CN})$ and the single-function list (SF). 
told precisely how these rules fit the three-pair, double-function list that he received as a part of the training. After studying the three rules for $30 \mathrm{sec}$, the subject was transferred to the following three-pair list: soccer-sofa, sofa-strawberry, strawberry-soccer. The correct word in each pair is italicized. However, the subject responded with the category name. None of these instances occurred in List $C$, the list to which the subject was subsequently transferred. After the first 30-sec study period, the subject was given four test trials (no feedback) on the three-pair list at a 4:2-sec rate. This was followed by another 30 -sec study period on the three rules, another block of four test trials, and so on. This was continued until the subject had responded correctly to all pairs on a block of four test trials, or until six test blocks had been completed.

Test. After the training, the subjects were transferred to List $C$ and informed that if they applied the rules to the new pairs in the 15-pair list they would be correct. The subjects responded with the category instances. Ten trials were given on List C, using a $4: 2$-sec rate and the anticipation method.

\section{Results and Discussion}

During the training, 4 of the 16 subjects failed to apply the three rules successfully on a block of four trials on the three-pair list. If these subjects are assigned a value of six blocks, the mean number of blocks required to produce successful responding on the three-pair list for the 16 subjects was just slightly over four (4.06). On the average, therefore, it required slightly over $2 \mathrm{~min}$ of distributed study time before the subject could apply the three rules consistently to the three pairs of instances.

Transfer performance on the 15-pair C list was very high. On the first trial, the mean number of errors was 2.81. Seven of the 16 subjects responded perfectly on the first trial, and 13 of the 16 subjects had at least one errorless trial on the 10 trials given. The mean number of errors on the 10th trial was .69 .

These data indicate that a subject can learn three contingencies among three category names and apply tilem with some success to new instances of the categories. Why then, did not the usual subject do this when he was faced with the 15-pair List $\bar{C}$ in Experiment 1? It has been noted that the first step in such learning would be to discover that three contingency rules could govern performance. It is difficult to believe that if these rules were discovered they would not be utilized. The problem, therefore, appeared to lie in the discovery. Experiment III was an attempt to make the discovery process easier. The critical change was to require the subject to respond with the concept names rather than with the concept instances. In addition, the rate of presenting the pairs was slowed on the grounds that it might not be possible for the subject to apply the rules when the rate was $2: 2 \mathrm{sec}$ as in Experiment I.

\section{EXPERIMENT III}

\section{Method}

Lists. There were four lists identified in terms of their pairings
Table 1

Mean Number of Errors on Each of the Eight Anticipation Trials

\begin{tabular}{lcccccccc}
\hline & \multicolumn{8}{c}{ Trials } \\
\cline { 2 - 9 } List & 1 & 2 & 3 & 4 & 5 & 6 & 7 & 8 \\
\hline CI & 6.4 & 6.7 & 6.5 & 6.7 & 5.5 & 5.4 & 5.5 & 5.0 \\
CC & 7.7 & 6.8 & 6.6 & 5.8 & 6.2 & 5.1 & 5.2 & 4.5 \\
NCI & 6.7 & 6.4 & 6.4 & 6.3 & 5.1 & 5.6 & 6.5 & 5.1 \\
NCC & 7.7 & 7.0 & 7.2 & 5.8 & 5.8 & 5.2 & 5.8 & 5.3 \\
\hline
\end{tabular}

being contingent $(C)$ or noncontingent $(\mathrm{NC})$, and in terms of the nature of the feedback. The $C$ list was the same as used in Experiment I, with two versions. In one case the feedback was the correct instance, just as in Experiment $I$. This list is identified as the CI List. In the other case, the concept name was given during the feedback interval (List CC). Thus, the feedback consisted of the three category names (sport, fruit, furniture). The two NC lists consisted of the same 15 words as those in Lists $\mathrm{CI}$ and $\mathrm{CC}$, but the pairing was such that no two members of the same category ever occurred together. This made it possible to use the category name in the feedback for one of the versions of this list. These two noncontingent lists are identified as List NCC (noncontingent, category name during feedback) and NCI (noncontingent, instance feedback). For all four lists the subject responded with the category name.

Procedure. The lists were presented for anticipation learning at a 3:3-sec rate. The subjects were not instructed about contingencies but were fully informed about feedback and how it applied to the instance. Other details were the same as for Experiment I. Twenty subjects were assigned to each of the four lists according to a block-randomized schedule. After one study trial, eight anticipation trials followed.

\section{Results and Discussion}

The mean numbers of errors on each trial for each list are shown in Table 1. As was true for Experiment I, it is clear that learning occurred, but at a very slow pace. The analysis, including trials as one variable, showed that the learning was reliable $(F=12.30)$, but the only other source of reliable variation was the Trials by Type of Feedback interaction, $F(7,532)=2.21, p<.05$. This interaction is small quantitatively, but an inspection of Table 1 will show that, whereas performance was somewhat better with instance feedback than with category feedback on the initial trials, there was a slight reversal of this relationship during the later trials. Since the interaction is largely due to the difference on the initial trials, we are inclined to believe that the category feedback on the initial trials required some performance adjustment by the subjects. In any event, we will not consider the matter further. The critical fact shown in Table 1 is that learning the double-function list involving three contingencies was not enhanced by presentation of the category names during the feedback interval.

A few subjects reported acquiring the contingency rules. Others with equally good scores did not perceive the contingencies. One subject who gave perfect performance on three trials claimed he had used the length of the words as his cue. There is no reason to doubt that some learning based on associative contingencies did occur, but the data clearly emphasize 
that the discovery and utilization of three contingencies does not occur with ease, however simple it may seem in the abstract.

\section{GENERAL DISCUSSION}

The data leave little doubt concerning a conclusion about contingent associations. Very few subjects will discover, learn, and utilize such contingencies even when a very difficult task could be sharply reduced in difficulty by doing so. Experiment II showed that most subjects can learn the three contingent associations when these associations are studied independently, although this was not an easy task when judged by-the comparable difficulty of learning three paired associates. Such evidence leads to the conclusion that the slow learning which does occur in the usual double-function list is not based upon contingent associations since as many contingencies as pairs would be necessary for complete learning to occur.

If the double-function list is thought of as presenting a problem-solving situation in which the subject seeks rules, the present data suggest that a search for contingency rules is not a common one for the usual undergraduate student. This may simply result from the fact that contingency learning as represented by the double-function list is not frequently required in school subjects. Or, it may be that contingency learning, for whatever reason, is simply difficult for the memory system to handle. The overlap of stimulus elements may be heavily involved in making the task a difficult one.

A quite different matter is involved in trying to understand how any learning of a double-function list occurs if the learning does not involve contingencies. Mueller et al. (1974) found that the learning could be facilitated somewhat when the subjects were asked to form a sentence or pirase incorporating both the correct and incorrect words in the pair. This finding, plus other evidence, led these investigators to suggest that the association which develops between the words in a pair is in some way responsible for the learning that is observed. As noted earlier, the present experiments were not intended to be analytical with regard to the basis of learning the double-function list except insofar as contingencies might have been involved. One set of relevant observations should be reported, however.

For each list in Experiment III, the difficulty of learning each pair was determined using the correct response (instance) to identify a pair. Of course, across all lists, each of the 15 words was a correct response in some pair. The 20 subjects assigned to each list were divided into two subgroups of 10 subjects each, and the number of errors made on each of the 15 pairs by each subgroup was determined and correlated, using the rank-order correlation. These correlations provide a measure of the reliability of item difficulty within a list. The four correlations varied from .62 to .82 , indicating appreciable agreement across subjects as to item difficulty. Furthermore, when correlations were calculated across lists, the agreement was substantial, with the six values ranging from .50 to .79 . It would seem, therefore, that whatever underlies the learning observed for double-function lists, idiosyncratic factors are not of great consequence.

\section{REFERENCES}

Ekstrand, B. R., Wallace, W. P., \& Underwood, B. J. A frequency theory of verbal-discrimination learning. Psychological Review, 1966, 73, 566-578.

Kausler, D. H., \& Boka, J. A. Effects of double functioning on verbal-discrimination learning. Journal of Experimental Psychology, 1968, 76, 558-567.

Mueller, J. H., Kausler, D. H., Yadrick, R. M., \& Pavur, E. J. Encoding strategies in double-function verbal-discrimination learning. Journal of Experimental Psychology: Human Learning and Memory, 1975 (in press).

Wickelgren, W. A. Context-sensitive coding, associative memory, and serial order in (speech) behavior. Psychological Review, $1969,76,1-15$.

\section{NOTE}

1. We wish to thank Ronald H. Nowaczyk for conducting this study.

(Received for publication August 23, 1974; Revision received September 7,1974 ,) 